\title{
The Relationship between Dental Indentation and Maxillary Sinusitis
}

\author{
Duzgun Yildirim ${ }^{1 \#}$, Murat Eroglu², Murat Salihoglu ${ }^{3}$, Ali Osman Yildirim², \\ Huseyin Karagoz ${ }^{4}$, Mustafa Erkan ${ }^{5}$ \\ ${ }^{1}$ Centermed Advanced Imaging Center, Department of Radiology, Istanbul, Turkey \\ ${ }^{2}$ Gulhane Military Medical Academy, Haydarpasa Training Hospital, Department of Emergency Medicine, Istanbul, Turkey \\ ${ }^{3}$ Gulhane Military Medical Academy, Haydarpasa Training Hospital, Department of Ear, Nose, Throat, Istanbul, Turkey \\ ${ }^{4}$ Gulhane Military Medical Academy, Haydarpasa Training Hospital, Department of Plastic Surgery, Istanbul, Turkey \\ ${ }^{5}$ Gulhane Military Medical Academy, Haydarpasa Training Hospital, Department of Orthodontics, Istanbul, Turkey \\ Email: \#yduzgun@yahoo.com
}

Received February 26, 2013; revised March 27, 2013; accepted April 12, 2013

Copyright (C) 2013 Duzgun Yildirim et al. This is an open access article distributed under the Creative Commons Attribution License, which permits unrestricted use, distribution, and reproduction in any medium, provided the original work is properly cited.

\begin{abstract}
Purpose: To determine the relationship between the dental root indentation and maxillary sinusitis. Methods: We assessed the records of the patients who underwent paranasal sinus computed tomography imaging for suspected sinusitis. Results: We identified a total of 52 patients with a pre-diagnosis of maxillary sinusitis. Dental indentation was detected in 58 of $104(55.7 \%)$ sinuses. Forty six of 58 sinuses (79.3\%) with dental indentation had mucosal thickening higher than $2 \mathrm{~mm}$. The difference between the groups was statistically significant $(\mathrm{p}=0.007)$. Conclusion: Dental indentation should be kept in mind as a reason for chronic maxillary sinus inflammation, if an underlying cause cannot be identified.
\end{abstract}

Keywords: Maxillary Sinus; Dental Indentation; Mucosal Thickening; Computerized Tomography

\section{Introduction}

Sinusitis is a multifactorial disease characterized by the inflammation of paranasal sinus mucosa due to viral, bacterial or fungal infections of upper respiratory tract [1]. The maxillary sinuses are located between the nasal, oral and orbital cavities and are therefore most susceptible of all sinuses for the invasion of pathogenic bacteria through the nasal ostium or the oral cavity. Since maxillary sinuses are in vicinity of vital structures, their infections should be treated promptly [2]. The most common maxillary sinus radiologic findings are mucosal thickening and mucosal cysts [3]. Mucosal thickening of maxillary sinus is twice as widespread in patients with dental disease as in the general population [4] and the reported prevalence of mucosal thickening in radiographic surveys ranges between $23 \%$ and $31 \%$ [5]. Additionally, it has been reported that mucosal thickening is detected at a higher rate on cross-sectional imaging methods like computerized tomography (CT) for MRI than X-rays [6].

The existence of mucosal thickening in the maxillary sinus generally presupposes a mucosal irritation [7].

\footnotetext{
${ }^{*}$ Conflict of interest: The authors have no conflicts of interest.

${ }^{\#}$ Corresponding author.
}

Odontogenic irritations include periodontal abscesses, nonvital maxillary teeth, retained roots, embedded teeth, extensively carious teeth, and oro-antral fistulae [8]. Odontogenic reasons such as periodontal disease and periapical lesions are reported to cause $58 \%$ to $78 \%$ of maxillary sinus mucosal thickening [3].

Radiologic imaging is an important tool in establishing the diagnosis. CT is helpful for evaluating the relationship of the maxillary teeth's roots with the maxillary sinus floors. CT reveals the presence of mucosal thickness, any displaced roots, teeth or presence of any foreign bodies inside the sinus boundaries [6]. The aim of this study was to investigate the relationship between the presence of dental indentation into maxillary sinus and mucosal thickening.

\section{Materials and Methods}

This retrospective study included patients which have undergone paranasal sinus $\mathrm{CT}$ imaging for suspected sinusitis. Study protocol was approved by Local Ethics Committee. Demographic data and past medical history of the patients were obtained from medical records. 
When all patients were retrospectively screened from the archive, they were consistent with sinusitis in respect symptomatologic and they were diagnosed as sinusitis with symptomology and after CT imaging, patients who have clinically and radiological confirmed sinusitis received medical treatment. On CT examinations, cases having primary dental pathologies (abscess, odontogenic tumor etc.) were excluded from the study. CT images and medical records of 52 consecutive patients visiting the Department of Ear, Nose and Throat were assessed.

Examinations were performed on a helical CT (Somatom DRH, Siemens, Erlengen, Germany). Adjustments of the sections were achieved on lateral scenograms. Axial and coronal CT scans with $5 \mathrm{~mm}$ slice thicknesses were available in each of the patients. Sections were reconstructed automatically and evaluated by an experienced radiologist. Axially obtained row data images were reconstructed to thin $(2 \mathrm{~mm}$ ) sectional (coronal and sagittal) images with using automatic sharp-bone algorithm. In each case horizontally oriented line has been drew which crosses tangentially the deepest point of the maxillary sinus floor (Figure 1). Maximal height of the related indentation of the maxillary tooth and the mucosal thickness recorded together in a table. The indentation degree was recorded and mucosal thickening of the maxillary sinus was defined as positive in the existence of soft tissue structures thickness $>2 \mathrm{~mm}$. Also the thickening and accompanying retention cyst was also noted.

Maxillary sinuses without tooth indentation of the same cases were used as a normal control group. All measures have been done using an accurate measuring tool (with an accuracy to the nearest $=0.1 \mathrm{~mm}$ ) in the software, by using a diagnostic display-screen.

Then, using all the recorded data, statistical analyses were performed to reveal the relationship between the dental root indentation and maxillary sinusitis. These statistical analyses have been made by using SPSS 11 for Windows (Chicago, IL). Data were presented as mean \pm SD. Categorical variables were analyzed by using ChiSquare test and continuous variables were analyzed by using independent samples $t$ test. A $p<0.05$ was considered as statistically significant.

\section{Results}

The study group consisted of 52 patients (50\% males and $50 \%$ females) with a mean age of $33.1+/-10.2$ years. Dental indentation was detected in 58 of $104(55.7 \%)$ sinuses. Mean length of dental indentation was $4.31 \pm$ $2.87 \mathrm{~mm}$. Dental indentation was bilateral in 20 (38.4\%) cases and unilateral in 19 (36.5\%) cases. Mucosal thickening higher than $2 \mathrm{~mm}$ was detected in 71 of 104 $(68.3 \%)$ sinuses. Forty six of 58 sinuses $(79.3 \%)$ with dental indentation and 25 of 46 (54.3\%) sinuses without dental indentation had mucosal thickening. The difference between the groups was statistically significant ( $p=$ 0.007). Furthermore, mucosal thickness was significantly higher in sinuses with dental indentation $(7.76 \pm 8.39$ $\mathrm{mm})$ compared to sinuses without dental indentation $(3.17 \pm 4.14 \mathrm{~mm})(\mathrm{p}=0.001)$.

\section{Discussion}

Maxillary sinuses develop earliest among sinuses. Its development starts in 3rd month of fetal life and ends at $14-16$ ages when alveolar development of upper teeth ends [9]. It is frequently seen that the roots of molar teeth perforates maxillary sinus floor which is formed during posterior development of the alveolar process of the maxilla. In addition, the maxillary teeth roots may protrude into the sinus cavity during continued expansion and pneumatization of the maxillary sinus $[10,11]$.

In general, incisor and canine teeth do not have contiguity with sinuses, but premolar and specifically molar teeth are located beneath the maxillary sinus floor. As a result of these close relationship, periapical infections might cause mucosal irritation and thickening within the

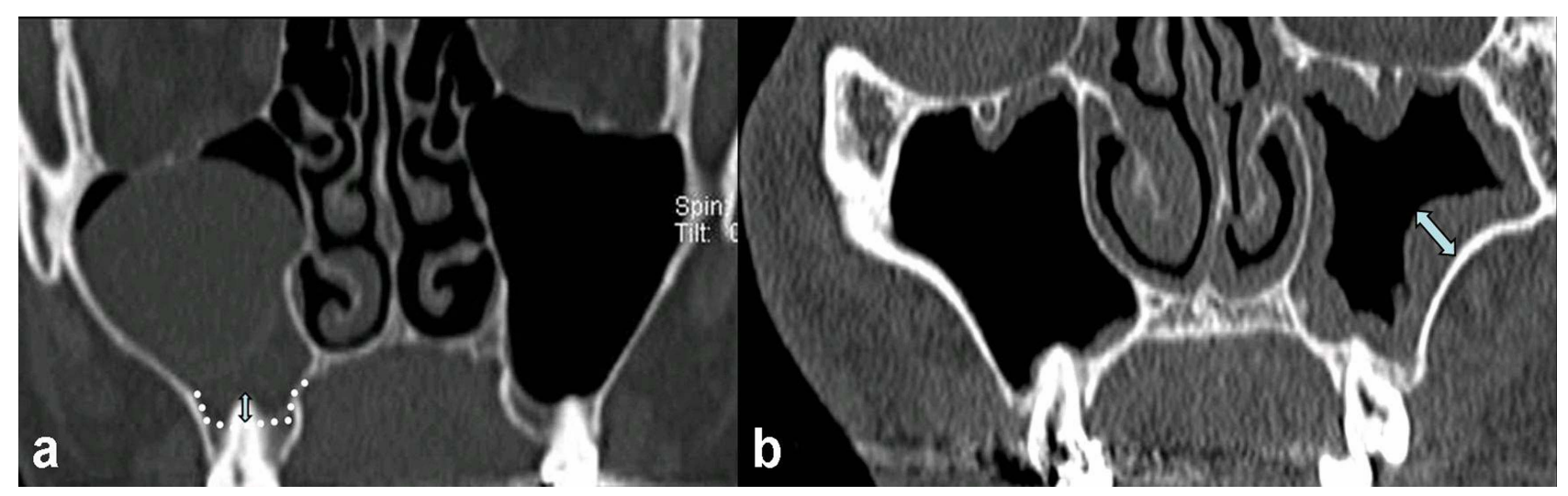

Figure 1. Coronal section paranasal sinus CT images of different cases. (a) Measurement of the indentation degree of the root represented by a bi-directional arrow which ascending the mucosal floor line (dashed line). (b) In another case, mucosal thickening was measured in the thickest part of the pathology (bi-directional arrow). 
maxillary sinus [4].

The floor of the sinus is an effective barrier that rarely allows for direct penetration of odontogenic infections into the maxillary sinus. However, especially in individuals whose dental roots are proximal to the floor of the sinus or protruded in to sinus cavity, odontogenic infections can drain into the sinus and this might result in reactive mucosal response within the sinus [4,12]. Vallo et al. suggested that pathologic dental findings and root canal treatments were significantly associated with mucosal thickening but not with mucosal cysts [3]. Results of the present study demonstrated a considerable difference in mean values of the mucosal thickening for the two groups of maxillary sinuses (with and without dental indentation).

The mucosal thickness of normal maxillary sinuses has been shown to be variable [13]. Some studies have suggested that, on average, the normal thickness of healthy sinuses can be approximately $1 \mathrm{~mm}$, with considerable variation among individuals [14]. In general, $2 \mathrm{~mm}$ was considered a reliable threshold for pathological mucosal swelling [15]. In our study, mucosal thickness over $2 \mathrm{~mm}$ was accepted as pathologic. We found that mucosal thickness was significantly higher in sinuses with dental indentation $(7.76 \pm 8.39 \mathrm{~mm})$ compared to sinuses without dental indentation $(3.17 \pm 4.14 \mathrm{~mm})(\mathrm{p}=0.001)$.

Although, there are studies investigating dental diseases and maxillary sinuses pathologies, there is not enough study investigating the relationship between dental indentation and maxillary sinusitis. Abrahams et al. demonstrated that a two fold increase occurred in maxillary sinus disease in patients with periodontal disease and suggested a causal relationship [10]. Falk et al. suggested that patients who undergo successful treatment of periodontal disease have a significant decrease in the incidence of maxillary sinus disease [16].

Radiological imaging is an important tool for establishing the diagnosis of sinus diseases $[17,18]$. The ability to visualize bone and soft tissue and obtain thin sections and multiple views makes the CT preferred imaging method to evaluate paranasal sinuses [12]. Axial and coronal views also allow visualizing periapical odontogenic abscess close to the sinus floor defect and diseased sinus tissue. CT should be preferred when clinical findings strongly suggest sinusitis but direct X-rays fails to detect sinusitis [19].

Sinus disease of odontogenic origin deserves special consideration because of some subtle differences in pathophysiology, microbiology, and management as compared with routine community-acquired sinusitis. If not recognized, the treatment of sinus disease will fail and cause prolonged medical therapy or unnecessary surgery $[1,4]$.

There were some limitations during this study. First of all, the evaluation of cases in terms of symptomatology performed by an Ear Nose Throat specialist and this was a subjective assessment. Secondly, we chose the value of $2 \mathrm{~mm}$, to get a certain thickness in each measurement, as well as to measure objectively in CT section. We chose this value because we had to set a minimum threshold value can be measured clearly.

\section{Conclusion}

We found a relationship between dental root indentation and mucosal thickening in maxillary sinuses. Therefore, dental indentation should be kept in mind as a reason for chronic maxillary sinus inflammation, if an underlying cause cannot be identified. A close collaborative effort between medical and dental specialists is essential for an accurate diagnosis and optimal treatment. Further multicentre studies are needed to confirm the relationship between dental indentation and maxillary sinusitis.

\section{REFERENCES}

[1] O. Arias-Irimia, C. Barona-Dorado, J. A. Santos-Marino, N. Martínez-Rodriguez and J. M. Martínez-González, "Meta-Analysis of the Etiology of Odontogenic Maxillary Sinusitis," Medicina Oral Patologia Oral y Cirugia Bucal, Vol. 15, No. 1, 2010, pp. 70-73.

doi:10.4317/medoral.15.e70

[2] D. P. Kretzschmar and J. L. Kretzschmar, "Rhinosinusitis: review from a dental perspective", Oral Surgery, Oral Medicine, Oral Pathology, Oral Radiology, and Endodontics, Vol. 96, No.2, 2003, pp. 128-135. doi:10.1016/S1079-2104(03)00306-8

[3] J. Vallo, L. Suominen-Taipale, S. Huumonen, K. Soikkonen and A. Norblad, "Prevalence of Mucosal Abnormalities of the Maxillary Sinus and Their Relationship to Dental Disease in Panoramic Radiography: Results from the Health 2000 Health Examination Survey," Oral Surgery, Oral Medicine, Oral Pathology, Oral Radiology, and Endodontics, Vol. 109, No. 3, 2010, pp. 80-87. doi:10.1016/j.tripleo.2009.10.031

[4] A. Whyte and G. Chapeikin, "Opaque Maxillary Antrum: A Pictorial Review," Australasian Radiology, Vol. 49, No. 3, 2005, pp. 203-213. doi:10.1111/j.1440-1673.2005.01432.x

[5] K. Patel, S. V. Chavda, N. Violaris and A. L. Pahor, "Incidental Paranasal Sinus İnflammatory Changes in a British Population," Journal of Laryngology and Otology, Vol. 110, No. 7, 1996, pp. 649-651. doi: $10.1017 / \mathrm{S} 0022215100134516$

[6] I. Brook, "Sinusitis of Odontogenic Origin," Otolaryngology-Head and Neck Surgery, Vol. 135, No. 3, 2006, pp. 349-355. doi:10.1016/j.otohns.2005.10.059

[7] H. M. Worth and D. W. Stoneman, "Radiographic İnterpretation of Antral Mucosal Changes Due to Localized Dental İnfection," Journal of the Canadian Dental Association, Vol. 38, No. 3, 1972, pp. 111-116.

[8] N. L. Rhodus, "The Prevalence and Clinical Significance 
of Maxillary Sinus Mucous Retention Cyst in a General Clinic Population," Ear, Nose and Throat Journal, Vol. 69, No. 2, 1990, pp. 82-90.

[9] H. C. Kelley and L. W. Kay, "The Maxillary Sinus and İts Dental İmplications," John Wright and Sons, Bristol, 1975.

[10] J. Abrahams and R. M. Glassberg, "Dental Disease: A Frequently Unrecognized Cause of Maxillary Sinus Abnormalities?" American Journal of Roentgenology, Vol. 166, No. 5, 1996, pp. 1219-1223.

[11] H. Sicher, "The Viscera of Head and Neck," CV Mosby, St. Louis, 1975.

[12] P. Mehra, A. Caiazzo and S. Bestgen, "Odontogenic Sinusitis Causing Orbital Cellulitis: A Case Report," Journal of the American Dental Association, Vol. 130, No. 7, 1999, pp. 1086-1092.

[13] C. Phillips and T. Platts-MilIs, "Chronic Sinusitis: Relationship between CT Findings and Clinical History of Asthma, Allergy, Eosinophilia, and İnfection", American Journal of Roentgenology, Vol. 164, No. 1, 1995, pp. 185-187.

[14] J. P. Van den Bergh, C. M. ten Bruggenkate, F. J. Disch and D. B. Tuinzing, "Anatomical Aspects of Sinus Floor Elevations," Clinical Oral Implants Research, Vol. 11, No. 3, 2000, pp. 256-265. doi:10.1034/j.1600-0501.2000.011003256.x

[15] C. A. Cagici, C. Yilmazer, C. Hurcan, C. Ozer and F. Ozer, "Appropriate İnterslice Gap for Screening Coronal Paranasal Sinus Tomography for Mucosal Thickening," European Archives of Otorhinolaryngology, Vol. 266, No. 4, 2009, pp. 519-525. doi:10.1007/s00405-008-0786-6

[16] H. Falk, S. Ericson and A. Hugosona, "The Effects of Periodontal Treatment on Mucosal Membrane Thickening in the Maxillary Sinus," Journal of Clinical Periodontology, Vol. 13, No. 3, 1986, pp. 217-222.

[17] K. Yoshiura, S. Ban, T. Hijiya, K. Yuasa, K. Miwa, E. Ariji, et al., "Analysis of Maxillary Sinusitis Using Computed Tomography," Dentomaxillofacial Radiolology, Vol. 22, No. 2, 1993, pp. 86-92.

[18] D. Yildirim, O. Saglam, B. Gurpinar and T. Ilica, "Nasal Cavity Masses: Clinico-Radiologic Collaborations, Differential Diagnosis by Special Clues," Open Journal of Medical Imaging, Vol. 2 No. 1, 2012, pp. 10-18. doi:10.4236/ojmi.2012.21002

[19] E. Konen, M. Faibel, Y. Kleinbaum, M. Wolf, A. Lusky, C. Hoffman, et al., "The Value of the Occipitomental (Water's) View in Diagnosis of Sinusitis: A Comparative Study with Computed Tomography," Clinical Radiology, Vol. 55, No. 11, 2000, pp. 856-860. 